\title{
Alkaliptosis: a new weapon for cancer therapy
}

\author{
Jiao Liu' ${ }^{1}$ Feimei Kuang ${ }^{1} \cdot$ Rui Kang ${ }^{2} \cdot$ Daolin Tang ${ }^{1,2}$
}

Received: 25 July 2019 / Accepted: 16 August 2019 / Published online: 30 August 2019

(c) Springer Nature America, Inc. 2019

\begin{abstract}
Malignant tumors are one of the major causes of death worldwide, and the development of better treatments is urgently needed. There are many types of cancer treatment, such as surgery, chemotherapy, radiation therapy, immunotherapy, and targeted therapy, that might improve patient outcomes in a genotype- and stage-dependent manner. The main goal of cancer therapy is to inhibit biological capabilities of tumors and eventually eliminate the cancer cells. However, cancer cells are well known to escape apoptosis, a form of programmed cell death that was first described in studies of cell development and tissue remodelling. Increasing our understanding of cell death may result in new anticancer approaches that target types of nonapoptotic cell death, such as necroptosis, ferroptosis, autophagy-dependent cell death, and alkaliptosis. Notably, alkaliptosis, a pH-dependent form of regulated cell death, has been recently identified as a new strategy for cancer therapy across multiple tumor types, especially in pancreatic cancer.
\end{abstract}

Cell death was first described in an investigation of metamorphosis by Vogt in 1842 and is now recognized as a key biological event in a variety of physiological and pathological conditions [1]. Cell death has traditionally been divided into type I (also known as apoptosis), type II (also known as autophagic cell death), and type III (also known as necrosis) based on morphological criteria and histologic changes [2]. The Nomenclature Committee on Cell Death recently updated its guidelines for the classification of cell death subroutines [3]. In these guidelines, cell death can be classified into two distinct forms: accidental cell death (ACD) or regulated cell death (RCD) [3]. ACD is an uncontrolled form of cell death, whereas RCD is a controlled cellular process that occurs with genetic and biochemical changes [3]. Understanding the molecular machinery of RCD has had an enormous impact on all areas of biomedicine, including cancer research [4].

Apoptosis is the most common form of RCD and plays an essential role in tissue development and homeostasis [5]. Caspases are central components of the intrinsic pathway

Daolin Tang

daolin.tang@utsouthwestern.edu

1 The Third Affiliated Hospital, Guangzhou Medical University, Guangzhou, Guangdong, China

2 Department of Surgery, UT Southwestern Medical Center, Dallas, TX, USA (also known as the mitochondrial pathway) and extrinsic pathway (also known as the death receptor pathway) of apoptosis although a caspase-independent pathway may occur in some conditions $[6,7]$. Most cytotoxic anticancer drugs (e.g., alkylating agents, platinum compounds, and topoisomerase inhibitors) kill cancer cells through the induction of caspase-dependent apoptosis [8]. However, cancer cells can acquire genetic changes leading to apoptosis resistance and subsequent therapy failure $[9,10]$. Thus, triggering apoptosisindependent RCD, such as necroptosis (a mixed-lineage kinase domain-like pseudokinase [MLKL]-dependent form of regulated necrosis) [11, 12], ferroptosis (an iron-dependent form of regulated necrosis) [13-15], autophagy-dependent cell death (an autophagy-related [ATG] protein-mediated cell death) [16, 17], and alkaliptosis (a pH-dependent form of regulated necrosis) [18] (Fig. 1), may serve as a potential strategy for anticancer drug development.

Pancreatic cancer, the seventh-leading cause of cancerrelated deaths worldwide, often develops resistance to current chemotherapy, and new efficient anticancer drugs are needed for it [19-21]. Pancreatic ductal adenocarcinoma (PDAC), the most common type of pancreatic cancer, is driven by an oncogenetic KRAS mutation [22]. G proteincoupled receptors (GPCRs), one of the largest membrane protein families, remain the main target for modern drug discovery [23]. To identify novel chemotherapeutic agents for PDAC, a GPCR drug library was tested in PANC1, a human PDAC cell line harboring the most common $K R A S^{G 12 D}$ mutation. This screen identified a GPCR-targeted 


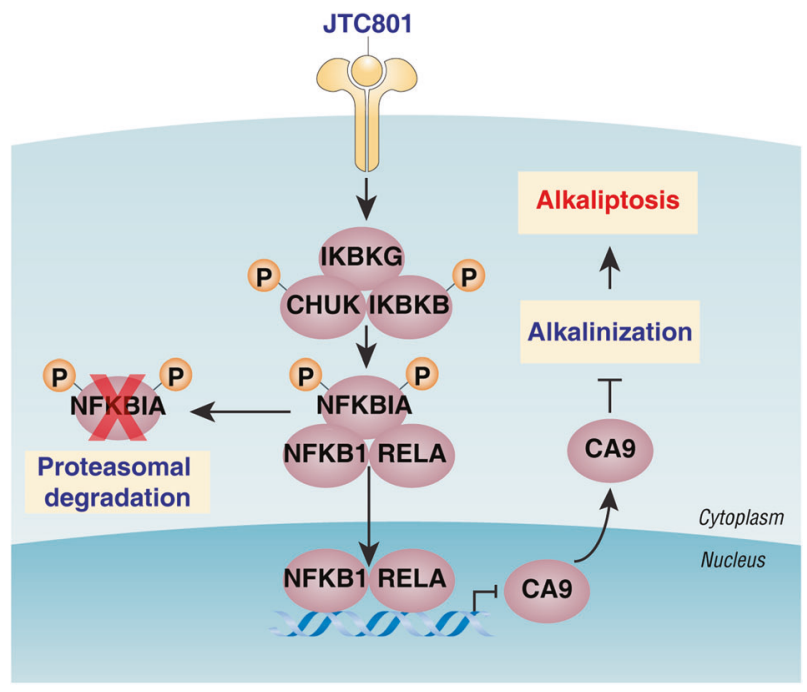

Fig. 1 The molecular mechanism of alkaliptosis. Alkaliptosis is driven by intracellular alkalinization after JTC801 activates the NF-кB pathway and subsequent CA9 downregulation

small molecule called JTC801 that significantly triggered cell death in multiple human PDAC cell lines, mouse pancreatic cancer-associated stellate cell lines, and primary human PDAC cells [18]. A National Cancer Institute-60 (NCI-60) screen using 60 different human tumor cell lines further confirmed the wide anticancer activity of JTC801 in kidney carcinomas, prostate cancer, melanoma, and central nervous system cancer cell lines in vitro. The oral administration of JTC801 suppressed tumor growth in xenograft, orthotropic, lung metastases, and genetically engineered mouse models of pancreatic cancer in vivo. Notably, toxicity from JTC801 was not observed in normal cells and tissues, indicating that JTC801 may be selectively lethal to tumor cells and absent of significant side effects.

The anticancer activity of JTC801 was shown to be due to the induction of alkaliptosis, but not apoptosis, necroptosis, ferroptosis, and autophagy-dependent cell death [18]. The pharmacological inhibition of apoptosis (using Z-VADFMK), necroptosis (using necrosulfonamide), ferroptosis (using ferrostatin-1), and autophagy (using hydroxychloroquine) had no effects on JTC801-induced alkaliptosis. Furthermore, the genetic modification of apoptosis $\left(\mathrm{Bax}^{-1-} / \mathrm{Bak}^{-/-}\right.$cells), necroptosis $\left(\mathrm{Ripkl}^{-/-}, \mathrm{Ripk}^{-/-}\right.$, and

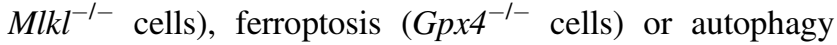
$\left(\mathrm{Atg}^{-1-}, \operatorname{Atg} 5^{-/-}, \operatorname{Atg} 7^{-1-}\right.$, and $\operatorname{Sqstm} 1^{-/-}$cells) also failed to block JTC801-induced alkaliptosis.

Much progress has been made in understanding how dysregulated $\mathrm{pH}$ affects cell survival and cell death. For example, intracellular acidification contributes to apoptosis [24], necroptosis [25], and autophagy [26]. In contrast, intracellular alkalinization is responsible for JTC801induced alkaliptosis and damage-associated molecular pattern (e.g., lactate dehydrogenase and high-mobility group box 1) release, which can be reversed by $N$-acetyl cysteine, $N$-acetyl alanine acid, and acidic medium (pH 6.2). Interestingly, oxidative stress is not essential for JTC801induced alkaliptosis. These findings demonstrate that alkaliptosis is an intracellular alkalinization-dependent form of regulated necrosis.

JTC801 has been shown to be a selective antagonist for opioid-related nociceptin receptor 1 (OPRL1), a nociceptin receptor distributed throughout the brain [27]. Although it is important for JTC801-induced analgesic function to reduce pain and anxiety [28-30], OPRL1 seems to be unimportant for JTC801-induced alkaliptosis [18]. Certain human tumor cell lines do not express OPRL1 but still are sensitive to alkaliptosis. OPRL1 depletion by RNAi fails to affect JTC801-induced alkaliptosis in OPRL1-expressed cancer cells. In addition, other OPRL1 antagonists (e.g., BANORL24, SB612111, UFP101, and Trap101) or a change of the binding of JTC801 to OPRL1 also has no influence on alkaliptosis. Surprisingly, kinase inhibitor screening uncovered that the nuclear factor $\kappa \mathrm{B}(\mathrm{NF}-\kappa \mathrm{B})$ pathway, a prosurvival pathway, is upregulated in many cancers and partly responsible for JTC801-induced alkaliptosis (Fig. 1) [18]. The canonical NF-кB pathway is activated by ligands (e.g., lipopolysaccharide) that leads to the recruitment and activation of an IKK protein complex, including components of the inhibitor of nuclear factor kappa B kinase complex (CHUK, also known as IKK $\alpha$ ), nuclear factor kappa B kinase subunit beta (IKBKB, also known as IKK $\beta$ ), and inhibitor of nuclear factor kappa $B$ kinase regulatory subunit gamma (IKBKG, also known as IKK $\gamma$ ) [31]. The IKK protein complex then phosphorylates and degrades NFKB inhibitor alpha (NFKBIA, also known as $I \kappa B \alpha$ ) leading to the subsequent nuclear translocation of nuclear factor kappa B subunit 1 (NFKB1, also known as p50) or RELA (also known as p65) to regulate gene expression. Blocking the NF- $\mathrm{KB}$ pathway by the pharmacological or genetic inhibition of IKBKB or RELA reduces JTC801-induced alkaliptosis in cancer cells. These findings raise the question of how the NF- $\mathrm{kB}$ pathway controls intracellular alkalinization. Indeed, carbonic anhydrase 9 (CA9), a member of the CA family that regulates $\mathrm{pH}$ by catalyzing the reversible hydration of carbon dioxide to carbonic acid, is identified as a NF- $\mathrm{kB}-$ dependent negatively regulated gene responsible for alkaliptosis inhibition. CA9 depletion not only recapitulates the NF- $\mathrm{\kappa B}$ pathwaymediated intracellular alkalinization phenotype, but also rescues alkaliptosis sensitivity in NF-kB-deficient cancer cells. Nevertheless, the findings also reinforce the notion that the transcription factor plays a dual role in mediating either cancer cell survival or cell death depending on its target genes and related regulatory networks.

Because dysregulated $\mathrm{pH}$ is a common feature of cancers, the manipulation of the extracellular and/or intracellular $\mathrm{pH}$ 
of tumors is a reasonable approach for cancer therapy [32]. In this regard, the discovery of alkaliptosis may hold promise by leading to cancer therapeutic targets to achieve higher specificity and efficacy. At present, nothing is known about the role of alkaliptosis in tumor development or about the effector molecule of plasma membrane rupture during alkaliptosis. Further clinical trials need to confirm the efficacy and safety of JTC801, although it may have two potential benefits for cancer patients: easing pain and anxiety symptoms and directly killing tumor cells via alkaliptosis.

Acknowledgements We thank Dave Primm (Department of Surgery, University of Texas Southwestern Medical Center) for his critical reading of the paper. JL is supported by grants from the National Natural Science Foundation of China $(31671435,81400132$, and 81772508).

\section{Compliance with ethical standards}

Conflict of interest The authors declare that they have no conflict of interest.

Publisher's note: Springer Nature remains neutral with regard to jurisdictional claims in published maps and institutional affiliations.

\section{References}

1. Clarke PG, Clarke S. Nineteenth century research on naturally occurring cell death and related phenomena. Anat Embryol (Berl). 1996;193:81-99.

2. Schweichel JU, Merker HJ. The morphology of various types of cell death in prenatal tissues. Teratology. 1973;7:253-66.

3. Galluzzi L, Vitale I, Aaronson SA, Abrams JM, Adam D, Agostinis P, et al. Molecular mechanisms of cell death: recommendations of the Nomenclature Committee on Cell Death 2018. Cell Death Differ. 2018;25:486-541.

4. Tang D, Kang R, Berghe TV, Vandenabeele P, Kroemer G. The molecular machinery of regulated cell death. Cell Res. 2019;29:347-64.

5. Elmore S. Apoptosis: a review of programmed cell death. Toxicol Pathol. 2007;35:495-516.

6. McIlwain DR, Berger T, Mak TW. Caspase functions in cell death and disease. Cold Spring Harb Perspect Biol. 2015;7:a026716.

7. Galluzzi L, Lopez-Soto A, Kumar S, Kroemer G. Caspases connect cell-death signaling to organismal homeostasis. Immunity. 2016;44:221-31.

8. Kaufmann SH, Earnshaw WC. Induction of apoptosis by cancer chemotherapy. Exp Cell Res. 2000;256:42-9.

9. Brown JM, Attardi LD. The role of apoptosis in cancer development and treatment response. Nat Rev Cancer. 2005;5:231-7.

10. Favrot M, Coll JL, Louis N, Negoescu A. Cell death and cancer: replacement of apoptotic genes and inactivation of death suppressor genes in therapy. Gene Ther. 1998;5:728-39.

11. Galluzzi L, Kepp O, Chan FK, Kroemer G. Necroptosis: Mechanisms and Relevance to Disease. Annu Rev Pathol. 2017;12:103-30.

12. Weinlich R, Oberst A, Beere HM, Green DR. Necroptosis in development, inflammation and disease. Nat Rev Mol Cell Biol. 2017;18:127-36.
13. Xie Y, Hou W, Song X, Yu Y, Huang J, Sun X, et al. Ferroptosis: process and function. Cell Death Differ. 2016;23:369-79.

14. Stockwell BR, Friedmann Angeli JP, Bayir H, Bush AI, Conrad M, Dixon SJ, et al. Ferroptosis: a regulated cell death nexus linking metabolism, redox biology, and disease. Cell. 2017;171: 273-85.

15. Zhou B, Liu J, Kang R, Klionsky DJ, Kroemer G, Tang D. Ferroptosis is a type of autophagy-dependent cell death. Semin Cancer Biol. 2019. pii: S1044-579X(19)30006-9. https://doi.org/ 10.1016/j.semcancer.2019.03.002. [Epub ahead of print].

16. Denton D, Kumar S. Autophagy-dependent cell death. Cell Death Differ. 2019;26:605-16.

17. Klionsky DJ, Abdelmohsen K, Abe A, Abedin MJ, Abeliovich H, Acevedo Arozena A, et al. Guidelines for the use and interpretation of assays for monitoring autophagy (3rd edition). Autophagy. 2016;12:1-222.

18. Song X, Zhu S, Xie Y, Liu J, Sun L, Zeng D, et al. JTC801 Induces $\mathrm{pH}$-dependent death specifically in cancer cells and slows growth of tumors in mice. Gastroenterology. 2018;154: $1480-93$.

19. Kleeff J, Korc M, Apte M, La Vecchia C, Johnson CD, Biankin AV, et al. Pancreatic cancer. Nat Rev Dis Prim. 2016;2:16022.

20. Bray F, Ferlay J, Soerjomataram I, Siegel RL, Torre LA, Jemal A. Global cancer statistics 2018: GLOBOCAN estimates of incidence and mortality worldwide for 36 cancers in 185 countries. CA Cancer J Clin. 2018;68:394-424.

21. Rawla P, Sunkara T, Gaduputi V. Epidemiology of pancreatic cancer: global trends, etiology and risk factors. World J Oncol. 2019;10:10-27.

22. di Magliano MP, Logsdon CD. Roles for KRAS in pancreatic tumor development and progression. Gastroenterology. 2013;144: 1220-9.

23. O’Hayre M, Vazquez-Prado J, Kufareva I, Stawiski EW, Handel $\mathrm{TM}$, Seshagiri S, et al. The emerging mutational landscape of $\mathrm{G}$ proteins and G-protein-coupled receptors in cancer. Nat Rev Cancer. 2013;13:412-24.

24. Furlong IJ, Ascaso R, Lopez Rivas A, Collins MK. Intracellular acidification induces apoptosis by stimulating ICE-like protease activity. J cell Sci. 1997;110(Pt 5):653-61.

25. Wang YZ, Wang JJ, Huang Y, Liu F, Zeng WZ, Li Y, et al. Tissue acidosis induces neuronal necroptosis via ASIC1a channel independent of its ionic conduction. eLife. 2015;4:e05682.

26. Nakamura N, Matsuura A, Wada Y, Ohsumi Y. Acidification of vacuoles is required for autophagic degradation in the yeast, Saccharomyces cerevisiae. J Biochem. 1997;121:338-44.

27. Yamada H, Nakamoto H, Suzuki Y, Ito T, Aisaka K. Pharmacological profiles of a novel opioid receptor-like1 (ORL(1)) receptor antagonist, JTC-801. Br J Pharmacol. 2002;135:323-32.

28. Bocheva A, Nocheva H, Pavlov N, Todorov P, Calmes M, Martinez J, et al. Synthesis and analgesic effects of novel beta2tryptophan hexapeptide analogs. Amino Acids. 2013;45:983-8.

29. Gear RW, Bogen O, Ferrari LF, Green PG, Levine JD. NOP receptor mediates anti-analgesia induced by agonist-antagonist opioids. Neuroscience. 2014;257:139-48.

30. Otsuka A, Doi H, Egawa G, Maekawa A, Fujita T, Nakamizo S, et al. Possible new therapeutic strategy to regulate atopic dermatitis through upregulating filaggrin expression. J Allergy Clin Immunol. 2014;133:139-46 e1-10.

31. Taniguchi K, Karin M. NF-kappaB, inflammation, immunity and cancer: coming of age. Nat Rev Immunol. 2018;18:309-24.

32. Webb BA, Chimenti M, Jacobson MP, Barber DL. Dysregulated $\mathrm{pH}$ : a perfect storm for cancer progression. Nat Rev Cancer. 2011;11:671-7. 